\title{
Correction to: Strategies of Drug Transporter Quantitation by LC-MS: Importance of Peptide Selection and Digestion Efficiency
}

\author{
Buyun Chen, ${ }^{1,3}$ Liling Liu, ${ }^{1}$ Hoangdung Ho, ${ }^{1}$ Yuan Chen, ${ }^{1}$ Ze Yang, ${ }^{2}$ Xiaorong Liang, ${ }^{1}$ Jian Payandeh, ${ }^{1}$ \\ Brian Dean, ${ }^{1}$ Cornelis E. C. A. Hop, ${ }^{1}$ and Yuzhong Deng ${ }^{1}$
}

Published online 6 June, 2018

\section{Correction to: The AAPS Journal}

https://doi.org/10.1208/s12248-017-0106-4

Liling Liu was not noted as the co-first author in the original article. Buyun Chen and Liling Liu contributed equally to the article.

The online version of the original article can be found at https://doi.org/ 10.1208/s12248-017-0106-4

${ }^{1}$ Genentech Inc, 1 DNA Way, South San Francisco, California, 94080, USA.

${ }^{2}$ Stanford University, 450 Serra Mall, Stanford, California, 94305, USA.

${ }^{3}$ To whom correspondence should be addressed. (e-mail: chen.buyun@gene.com) 